La meilleure définition d'un domaine émergent comme la biologie de synthèse est épistémique, c'est-à-dire qu'elle repose sur la collection de ce que la communauté scientifique s'entend à reconnaître comme des success stories. Cette définition est très ouverte, et ne saurait donc servir de support à la réglementation, notamment en matière de propriété intellectuelle.

Commençons par une première définition assez brutale. La biologie des systèmes est la science de l'analyse systémique des comportements dynamiques et spatiaux et des réseaux d'interaction entre biomolécules. La biologie de synthèse est un domaine émergent qui vise à la conception rationnelle, l'ingénierie ou la réingénierie de systèmes complexes fondés sur le vivant ou inspirés par le vivant, et dotés de fonctions absentes dans la nature. Cette ingénierie vise des applications utiles, conformément à la définition adoptée par le réseau de l'Espace européen de recherche de biologie de synthèse.

Si la biologie des systèmes permet de mieux comprendre les principes de design mis en œuvre dans la nature, la biologie de synthèse permet de les exploiter : la biologie des systèmes est analytique alors que la biologie de synthèse est synthétique. Cette définition reste néanmoins sujette à caution et nécessite d'être raffinée.

\section{Conditions scientifiques et technologiques de l'émergence de la biologie de synthèse}

François Képès

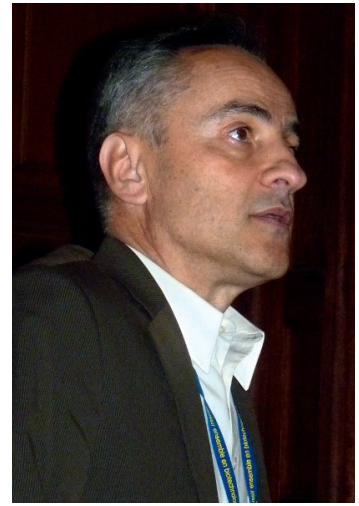

Institut de biologie systémique et synthétique de Genopole, CNRS FRE 3561,

Genopole Campus 1,

Genavenir 6,

5 , rue Henri Desbruères, 91030 Évry Cedex, France. francois.kepes@ epigenomique.genopole.fr
La biologie de synthèse ne se réduit pas à la biologie appliquée, mais permet de répondre efficacement à des interrogations d'ordre fondamental. Ainsi, l'implémentation d'un code génétique modifié dans une cellule vivante permet de déterminer si la quasi-universalité du code ADN tient à des contraintes physicochimiques, ou résulte d'un accident de l'évolution advenu à l'ancêtre de toutes les formes modernes de vie. On sait désormais que le code génétique ne résulte pas d'une contrainte physicochimique.

La biologie de synthèse recouvre trois démarches principales.

- La première relève d'une approche constructiviste et hiérarchique : elle part de «bio-briques », segments d'ADN aux fonctions définies, pour les assembler dans des dispositifs - circuits de régulation ou métaboliques - à l'instar des composants d'un circuit électronique. Ces dispositifs sont eux-mêmes assemblés dans des systèmes à l'échelle de la nanoparticule ou du micro-organisme.

- La deuxième approche, déconstructiviste, part d'un micro-organisme fonctionnel pour en retirer une partie du génome et y ajouter le codage du chemin métabolique d'intérêt, par exemple pour produire un vaccin ou un médicament. L'expérience la plus poussée en la matière a consisté à réaliser par synthèse chimique plus d'un million de paires de base, puis à introduire le chromosome ainsi synthétisé dans une bactérie capable de se diviser. II n'existe en revanche à ce stade aucun procédé de conception rationnelle de l'épigénome, alors que ce dernier recèle toute l'information contenue dans le vivant hors du génome. 


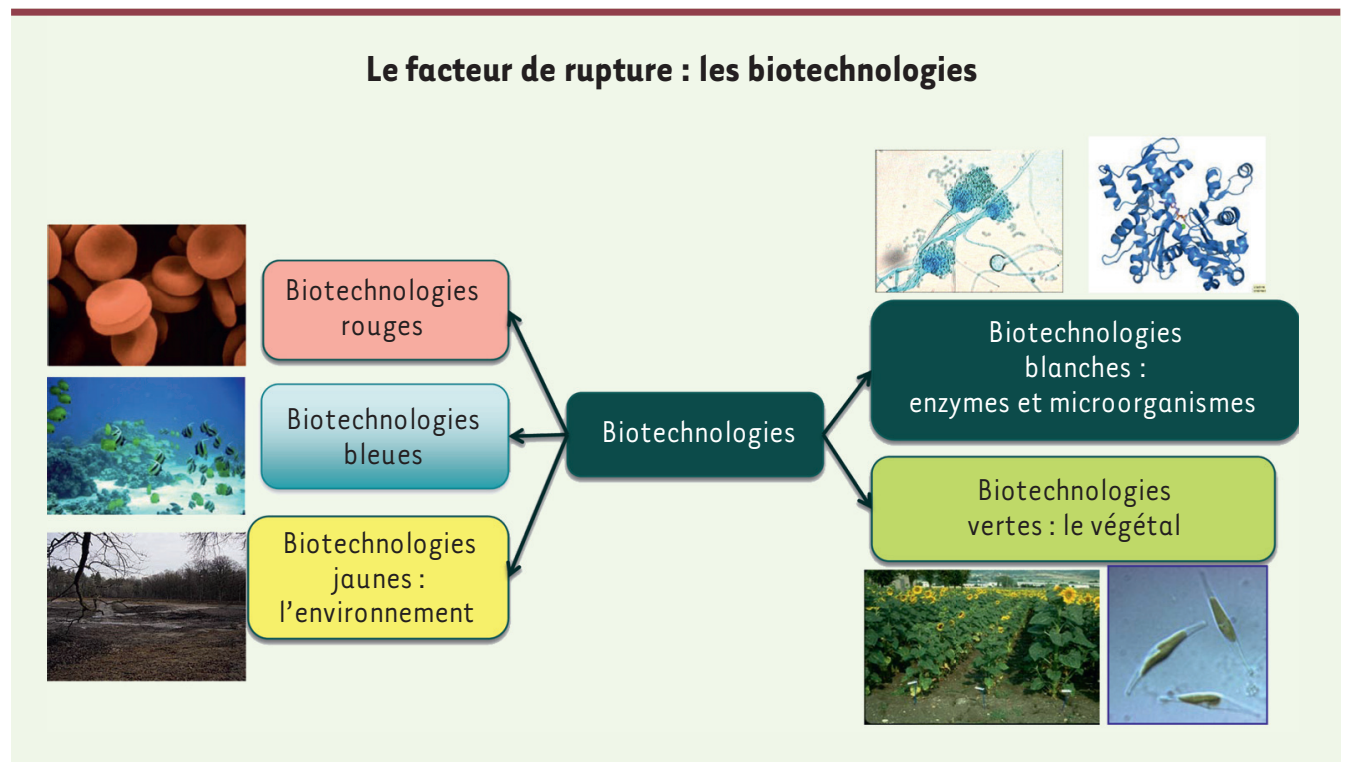

Figure 1. Les biotechnologies. ( ) Avec l'aimable autorisation de Paul Colonna.

- La troisième approche concerne les systèmes distincts du vivant trouvés dans la nature, et les systèmes protocellulaires. On peut par exemple modifier le code génétique, et plus tard il pourrait devenir possible de modifier le code de l'expression génique. Chimie de synthèse et biologie de synthèse se rencontrent dans la chimie innovante d'acides nucléiques ou d'acides aminés nouveaux. Enfin, les protocellules sont des liposomes plus sophistiqués observables dans des chambres microfluidiques: ces bulles lipidiques renferment un compartiment aqueux qui peut accueillir de nouveaux composants, et notamment des protéines. Le liposome peut par exemple cibler des cellules tumorales pour leur délivrer un produit toxique. Une application évidente est la réduction des effets secondaires d'un médicament pour accroître ses effets primaires. Sur un plan plus fondamental, le procédé permet d'observer les propriétés du vivant - croissance et division - dans des conditions simplifiées. Enfin, la notion de protocellule renvoie non seulement à la cellule simplifiée, mais aussi à la cellule primordiale, aux origines de la vie.

La biologie de synthèse renvoie donc à plusieurs mots clefs: hiérarchie de construction, modularité et normalisation, orthogonalité des systèmes et découplage entre conception et fabrication. Ce vocabulaire est familier des ingénieurs, mais est novateur quand il s'agit de biologie.

Le champ des applications est vaste; il recouvre les biotechnologies dites vertes, rouges et blanches (Figure 1). L'OCDE (Organisation de coopération et de développement économiques) décrit la biologie de synthèse comme "l'application de la science et de la technologie à des organismes vivants, de même qu'à ses composantes, produits et modélisations, pour modifier des matériaux vivants ou non vivants aux fins de la production de connaissances, de biens et de services ». La biologie de synthèse n'offre pas encore les caractéristiques d'une nouvelle discipline, notamment sur le plan institutionnel, mais peut être qualifiée de domaine émergent. Elle offre un large socle conceptuel et méthodologique, dont témoigne la variété de ses applications. L'humain tente d'acquérir une maîtrise croissante de son biotope; la biologie de synthèse n'est qu'une étape supplémentaire dans cette démarche.

L'analogie avec la chimie de synthèse est ici éclairante. Après le triomphe de la chimie analytique dans les années 1850 , les chimistes analyticiens se sont tournés vers la synthèse pour obtenir plus de produits que la nature n'en offre et ainsi mieux les étudier. La synthèse a donc d'abord porté sur les produits naturels, puis un petit pas a suffi pour synthétiser des produits non naturels. La recherche a débouché sur le développement, puis l'innovation, l'industrialisation, l'économie et l'emploi, donnant naissance à l'industrie chimique et la pétrochimie du $x x^{e}$ siècle. Par analogie, les années 1990 ont vu le triomphe de la biologie analytique au travers des «-omiques», filles de la biologie moléculaire: génomique, transcriptomique, etc. La biologie des systèmes, qui prévalait dans les années 1930, suscite alors de nouveau l'intérêt et attire des physiciens et des informaticiens. Elle se nourrit dans son analyse de données plus abondantes qu'auparavant. Quelques années plus tard apparaît la biologie de synthèse, qui s'appuie sur la compréhension ainsi développée.

La biologie de synthèse se nourrit de plusieurs disciplines et des sciences de l'ingénieur (Figure 2). L'augmentation des capacités de calcul et de stockage, l'amélioration de la modélisation mathématique et des simulations numériques et informatiques, ainsi que les avancées de l'intelligence artificielle et des algorithmes 


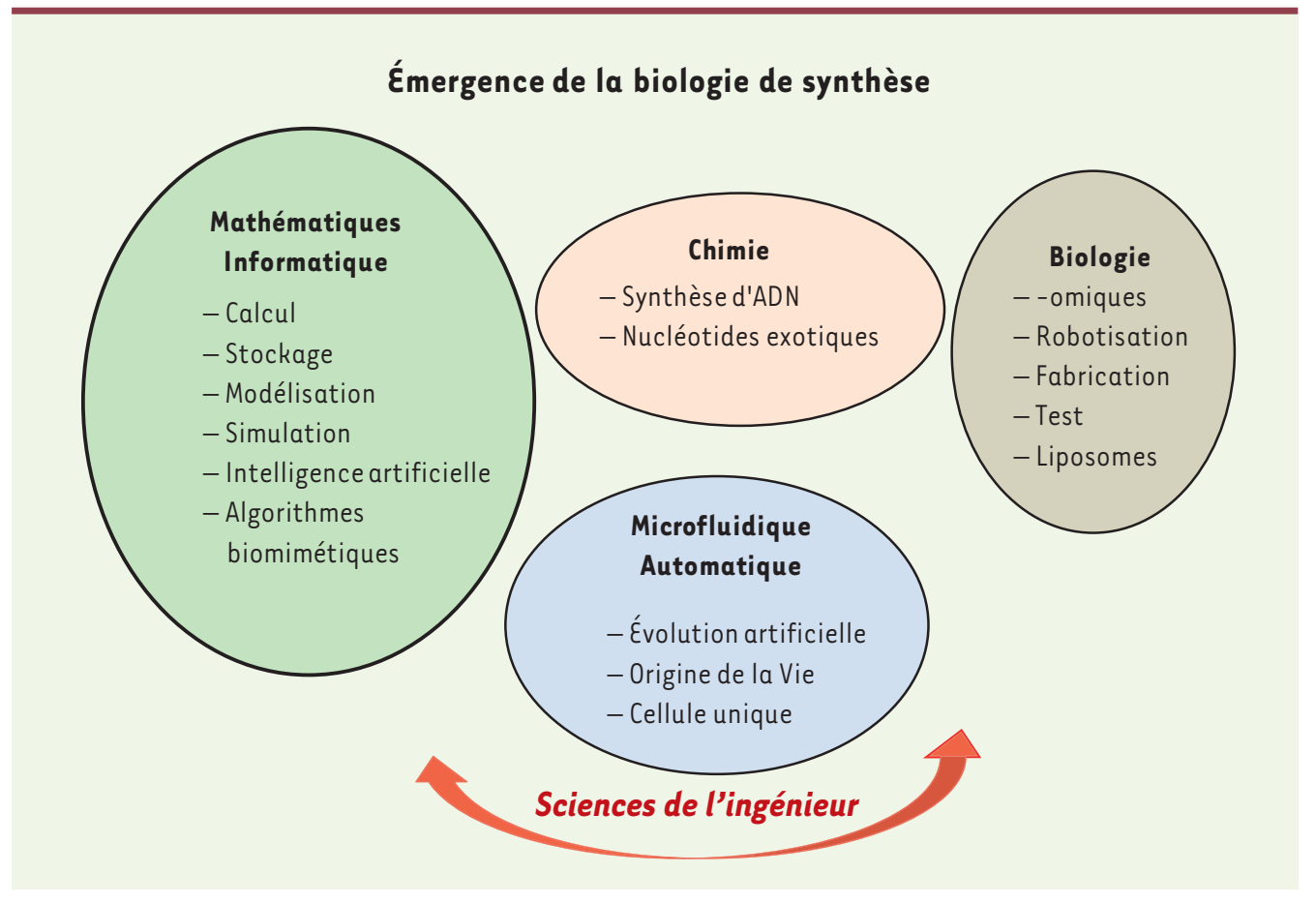

Figure 2. La biologie de synthèse est à la frontière de plusieurs disciplines et s'appuie sur les sciences de l'ingénieur.

biomimétiques, contribuent à la montée en puissance de la biologie de synthèse.

Du côté de l'automatique, théorie du contrôle et microfluidique permettent de mettre en œuvre l'évolution artificielle et de renouveler l'approche de l'origine de la vie. La microfluidique permet en particulier de travailler sur des cellules uniques et d'accéder à l'analyse de la variabilité des cellules plutôt qu'à celle de la moyenne d'une population.

Du côté de la chimie, la synthèse de l'ADN et la constitution de nucléotides exotiques sont des lieux privilégiés de synergie entre la chimie et la biologie de synthèse. Du côté de la biologie, la synergie joue à plein avec les «-omiques»: nous avons acquis la capacité de robotiser l'expérimentation et la rendre plus fiable pour fabriquer et tester des circuits biochimiques. D’importants progrès ont également été enregistrés pour l'autre expression moléculaire de la biologie de synthèse - les protocellules ou liposomes.

Les sciences de l'ingénieur irriguent l'ensemble. Le facteur le plus important dans l'émergence de la biologie de synthèse n'est pas tant l'arrivée de nouvelles capacités qu'un changement d'état d'esprit, qui a produit un effet boule de neige considérable. Les premiers échecs ont été lourds de succès futurs: ils ont permis de recalibrer les modèles, d'améliorer la compréhension fondamentale et de devenir de meilleurs ingénieurs. L'imagination est revenue au pouvoir grâce à ce nouveau domaine. $\diamond$

\section{LIENS D'INTÉRÊT}

L'auteur déclare n'avoir aucun lien d'intérêt concernant les données publiées dans cet article.

\section{TIRÉS À PART}

F. Képès 